\title{
Significance of lymph vessel invasion identified by the endothelial lymphatic marker D2-40 in node negative breast cancer
}

\author{
Angel Arnaout-Alkarain ${ }^{1}$, Harriette J Kahn², Steven A Narod ${ }^{3}$, Ping A Sun ${ }^{3}$ and \\ Alexander N Marks ${ }^{2,4}$ \\ ${ }^{1}$ Department of Surgery, Sunnybrook Health Sciences Centre and Women's College Hospital, University of \\ Toronto, Toronto, ON, Canada; ${ }^{2}$ Department of Pathology, Sunnybrook Health Sciences Centre and Women's \\ College Hospital, University of Toronto, Toronto, ON, Canada; ${ }^{3}$ Centre for Research in Women's Health, \\ University of Toronto, Toronto, ON, Canada and ${ }^{4}$ Banting \& Best Department of Medical Research, University \\ of Toronto, Toronto, ON, Canada
}

\begin{abstract}
Monoclonal antibody D2-40, a marker of lymphatic endothelium, identifies tumor emboli in lymph vessels. The aim of the study was to assess whether D2-40 + Iymph vessel invasion (LVI) correlates with clinicopathologic factors including lymphovascular invasion (LVI) as assessed by haematoxylin and eosin-stained sections (H\&E + or H\&E-) and to assess the prognostic significance in node-negative breast cancer. The study group consisted of 303 node-negative breast cancer patients that had a median follow-up of 7.6 years. Clinical and pathological data were retrieved from the Henrietta Banting database. Immunohistochemical staining was performed on formalin-fixed, paraffin-embedded tissue sections of the primary invasive carcinoma using D2-40. Immunostaining with CD31 was performed on the discordant cases that were H\&E + /D2-40-. D2-40 + Iymph vessel invasion was detected in $82 / 303(27 \%)$ cases. The foci of lymphatic invasion occurred predominantly at the invasive front of the tumor. The absence of D2-40 and CD31 in 13/17 discordant cases was suggestive of retraction artefact. D2-40 + lymph vessel invasion correlated significantly with age $(P=0.0003)$, tumor size $(P=0.005)$, histological grade $(P=\mathbf{0 . 0 0 0 1}), \mathrm{H} \& \mathrm{E}+(P=<\mathbf{0 . 0 0 0 1})$ and estrogen receptor status $(P=0.005)$ but not with histological type or progesterone receptor status. Multivariate analysis revealed that $\mathrm{D} 2-40+1 \mathrm{mmph}$ vessel invasion was the only significant predictor of distant recurrence. There was no significant association between D2-40 status and local recurrence $(P=0.752)$ or regional recurrence $(P=0.13)$. Both $\mathrm{D} 2-40+$ lymph vessel invasion $(P=0.009)$ and $H \& E+L V I$ cases $(P=0.02)$ were associated with overall shorter survival in univariate analysis. These data indicate that D2-40 identifies lymphatic invasion in breast tumors and is a significant predictor of outcome in breast cancer.
\end{abstract}

Modern Pathology (2007) 20, 183-191. doi:10.1038/modpathol.3800728; published online 5 January 2007

Keywords: node negative breast cancer; lymph vessel invasion; D2-40

The presence of axillary nodal metastasis is one of the most important prognostic factors in breast cancer. Patients with lymph node involvement have an increased risk of disease recurrence and reduced overall survival. ${ }^{1}$ However, the recurrence rate in patients that are axillary lymph node-negative ranges from $16 \%$ to $40 \% .^{2-4}$ Lymphovascular invasion (LVI), as shown by the presence of tumor cells

Correspondence: Dr HJ Kahn, MD, Department of Pathology, Sunnybrook Health Sciences Centre and Women's College Hospital, University of Toronto, E-433, 2075 Bayview Avenue, Toronto, ON, Canada M4N 3M5.

E-mail: harriette.kahn@sunnybrook.ca

Received 6 July 2006; revised 11 October 2006; accepted 19 October 2006; published online 5 January 2007 in blood vessels and/or lymphatic channels, is a required histopathological finding in assessment of primary breast tumors. Some studies have shown that LVI is an indicator of increased risk of recurrence and of reduced overall survival. ${ }^{2-9}$ LVI has also been shown to be a predictor of axillary lymph node metastasis..$^{7,10-13}$ It has been proposed that LVI could be used to identify a subgroup of axillary node-negative patients with an unfavorable prognosis that are likely to benefit from adjuvant chemotherapy. ${ }^{6-8}$ Furthermore the presence of peritumoral LVI, as assessed on haematoxylin and eosin- (H\&E) stained slides, has recently been added to the St Gallen criteria for the selection of adjuvant systemic therapy in operable breast cancer. ${ }^{14}$ 
The frequency of LVI ranges from $8 \%$ to $21 \%$ in lymph node-negative breast cancer patients ${ }^{2,3,6-8,15}$ and from $30 \%$ to $46 \%$ in lymph node-positive patients. ${ }^{7,8}$ The wide range of frequencies reported may be due to the difficulties of evaluation of LVI H\&E-stained sections of the tumor whereby LVI is identified by presence of tumor emboli within vascular channels distinctly lined by a single layer of endothelial cells $(\mathrm{H} \& \mathrm{E}+) .{ }^{15,16}$ This technique is subject to significant interobserver variability. ${ }^{9,15-17}$ The Eastern Cooperative Oncology Group reported that agreement in the identification of LVI in primary breast tumors by three pathologists occurred in only 12 out of 35 cases $(34.2 \%){ }^{16}$ Difficulty arises in the visualization of lymphovascular invasion on H\&E-stained slides for the following reasons. Firstly, tumor emboli that completely obliterate the lumen of a vessel may be difficult to discern. Secondly, retraction artifacts that isolate tumor aggregates and form as a result of tissue shrinkage during fixation may be indistinguishable from true tumor emboli in the lymphovascular space. In addition, it may be difficult to distinguish between blood vessel and lymph vessel invasion using H\&E staining.

The development of selective immunohistochemical markers for staining the vascular endothelium has increased the accuracy of detection of lymphovascular invasion in tumors. The majority of these immunohistochemical markers, including CD31, CD34 and Factor VIII-related antigen or Von Willebrand Factor, are selective for blood vessels, whereas staining of lymphatic channels by these markers has been either weak or negative. ${ }^{18-20}$ Antibodies identifying lymphatic endothelium have recently become available. VEGFR-3, a glycosylated tyrosine kinase cell surface receptor, has been used to stain lymph vessels but is also widely expressed in intratumoral blood vessels resulting in loss of specificity for lymphatics in tumors. ${ }^{21,22}$ LYVE-1, a specific receptor for hyaluronan, is expressed in endothelium of lymphatics. ${ }^{23}$ However, it is also expressed in some blood vessels of the lung and in hepatic sinusoids. ${ }^{23-25}$ Prox-1, a homeobox gene product is expressed in lymphatic endothelium throughout lymphatic development but its use in the immunohistochemical analysis of human neoplasms has been limited. ${ }^{26}$ Podoplanin, a membrane glycoprotein originally detected on glomerular podocytes, ${ }^{27}$ has been demonstrated in lymphatic endothelium and identified lymphatic invasion in cervical and breast cancers. ${ }^{28,29}$

Members of our research group recently reported that monoclonal antibody, D2-40, raised against a Mr40 000 membrane sialomucin, stained the endothelium of lymph vessels but did not react with the endothelium of capillaries, arteries and veins in normal and neoplastic tissues on formalin-fixed paraffin-embedded tissues. ${ }^{30-31}$ Furthermore D2-40 has been shown to react with the glycosylated and non-glycosylated epitopes of gp36, and is identical to podoplanin. ${ }^{32}$ D2-40 and podoplanin have been shown to be the most sensitive and specific markers for lymph vessel endothelium. ${ }^{33}$ We observed that D2-40 identified lymph vessel invasion in paraffinembedded sections of primary carcinomas of breast, colon, prostate, cervix and endometrium as well as melanomas. ${ }^{34,35}$ In a pilot study of 50 breast cancers, D2-40 increased the detection of lymph vessel invasion by $20 \%$ in node-negative cases and $16 \%$ in node-positive cases. ${ }^{34}$ Van den Eynden et al's ${ }^{36}$ study also showed that D2-40 distinguished lymphatic invasion from blood vessel invasion in breast cancers. In the present study, we correlated the presence of lymph vessel invasion identified by D2-40 with the clinicopathological factors in primary node-negative breast cancers. We also assessed the prognostic significance of lymph vessel invasion identified by D2-40 in these patients.

\section{Methods}

\section{Patient Eligibility and Clinical Data Accrual}

The study population consisted of 303 female patients with invasive, histologically proven lymph node-negative breast cancer, who had undergone surgery between the years 1989 to 1999 and had formalin-fixed paraffin-embedded blocks of the primary tumor available for further evaluation. The demographic, clinical and histopathological information of the patients were obtained from the Henrietta Banting Database of Sunnybrook and Women's College Health Sciences Centre (SWCHSC). The data included age at diagnosis, histological subtype, tumor size, histological grade (modified Bloom and Richardson), nuclear grade, LVI by H\&E, estrogen and progesterone status. Patients were treated with either modified radical mastectomy or local tumor resection with axillary lymph node dissection followed by breast irradiation in some cases. Adjuvant therapy with chemotherapy and/or hormonal therapy was given based on hormone receptor status and pathological grade of the tumor. Outcome data assessed included local, regional and distant recurrence as well as death.

\section{Immunohistochemistry}

H\&E-stained slides of the primary tumor were reviewed and one block with tumor and adjacent benign peritumoral tissue was selected for immunohistochemical staining. Sections $(5 \mu \mathrm{m})$ were cut from the formalin-fixed paraffin embedded blocks. One section was stained with H\&E. For D2-40 immunohistochemical analysis, $5 \mu \mathrm{m}$ sections of the paraffin-embedded tissues were first incubated in methanol containing $3 \% \mathrm{H}_{2} \mathrm{O}_{2}$ to inactivate endogenous peroxidase. The sections were subsequently incubated with $\mathrm{D} 2-40(0.1 \mu \mathrm{g} / \mathrm{ml})$, followed 
sequentially by biotinylated goat anti-mouse immunoglobulin (IgG) antibody (Zymed, San Francisco, CA, USA) at a 1:200 dilution and a horseradish peroxidase-avidin conjugate (Dako, Carpinteria, CA, USA) at a 1:500 dilution. For color development, the sections were incubated with $3,3^{\prime}$ diaminobenzidine. Immunohistochemical staining using antibody to CD31 (Clone JC/70A, Dako Carpetera, CA, USA) (as described previously) was subsequently performed on the subset of H\&E + /D2-40- cases. Cases that showed vascular invasion were categorized as $\mathrm{CD} 31+$.

\section{Assessment of Lymphatic Invasion}

The results of LVI from evaluation of the original surgical pathology reports was documented for the study. In addition, the $\mathrm{H} \& \mathrm{E}$ section of each case was assessed for LVI and were designated as H\&E + or H\&E-. The D2-40-stained slide was assessed for lymph vessel invasion without knowledge of the LVI status based on the H\&E slide or the original reports. A lymph vessel that showed positive staining of the endothelium for D2-40 and surrounded the tumor cells was diagnosed as positive for lymphatic invasion. The cases were categorized as $\mathrm{D} 2-40+$ or D2-40-. Interpretation of immunohistochemical results was made without knowledge of clinical outcome and the status of other prognostic variables.

\section{Statistical Methods}

$\chi^{2}$ analysis was used to compare the relationship between prognostic factors and lymph vessel invasion. Rates of distant recurrence and of overall survival curves were compared using the KaplanMeier method. Differences in the curves were evaluated for significance using the Log-rank test. The prognostic significance of $\mathrm{D} 2-40+$ lymph vessel invasion was assessed using univariate and multivariate Cox Proportional Hazards analysis. The prognostic significance of the subgroups, $\mathrm{H} \& \mathrm{E}-1$ $\mathrm{D} 2-40+v s \mathrm{H} \& \mathrm{E}+/ \mathrm{D} 2-40+$ and subgroups H\&E + / D2-40- vs H\&E-/D2-40 - were also evaluated to assess whether they had similar rates of outcome. Differences between groups were considered statistically significant if the $P$-value was less than 0.05 . All calculations were performed using the SAS statistical package.

\section{Results}

The mean age at diagnosis was 55.5 years (range 26.6-89.7 years) and the mean follow-up period was 7.38 years. The demographic, clinical, and histopathological characteristics of the 303 patients are shown in Table 1. Eighty-five $(28.1 \%)$ patients had surgery alone, 158 patients (52.1\%) received surgery and radiotherapy, 14 patients $(4.6 \%)$ had surgery and chemotherapy whereas 35 patients $(11.6 \%)$ had surgery, chemotherapy and radiotherapy. No information on chemotherapy or radiation was available on 11 patients $(3.6 \%)$. Seventy-six patients $(25 \%)$ experienced recurrences. Of these patients, 25 (8\%) had local recurrence, eight $(3 \%)$ had regional recurrence, and $43(14 \%)$ had distant recurrences. The total number of deaths within the follow-up period was $49(16.2 \%)$.

D2-40 stained the endothelium of lymph vessels but not the endothelium of capillaries, arteries or veins (Figure 1). Furthermore, D2-40 staining showed that very few lymph vessel were present within the tumor mass and most of the lymphatic channels were peritumoral (Figure 2). Lymph vessel invasion was identified by the presence of tumor emboli in vessels that showed D2-40 positivity of the endothelium. Figure 3 shows the presence of tumor emboli in lymph vessel that stained positively for D2-40. These emboli could easily be identified on H\&E as there was no obliteration of the lumen. Figure 4 shows a D2-40 + lymph vessel that contains a tumor embolus that completely obliterated the lumen of the lymphatic channel. This embolus could not be visualized on H\&E sections. D2-40 + lymph vessel invasion was mainly seen at the invasive front of the tumor. Although D2-40 was positive in myoepithelial cells of breast ducts and in occasional stromal cells in some cases, these could easily be differentiated from lymphatic channels based on their morphology.

LVI from the original reports that evaluated all the slides was identified in 53/303, whereas evaluation of the subsequent H\&E slide showed LVI in 52 of these cases $(\mathrm{H} \& \mathrm{E}+)$. D2-40 + lymph vessel invasion was identified in 82 of 303 patients (27\%). As shown in Table 1, the presence of D2-40+ lymph vessel invasion correlated with young age $(P=0.0003)$, large tumor size $(P=0.005)$, high tumor grade $(P=0.0001)$, high nuclear grade $(P=0.007)$, and estrogen receptors $(P=0.005)$. No association was seen with tumor histology $(P=0.06)$ or progesterone receptor status $(P=0.09) . \mathrm{D} 2-40+$ lymph vessel invasion strongly correlated with $\mathrm{H} \& \mathrm{E}+$ $(P=<0.0001)$. However, $46(18.4 \%)$ patients whose tumors were $\mathrm{H} \& \mathrm{E}$ - showed D2-40 + lymph vessel invasion. Immunohistochemical staining with D240 revealed that in 40 of these 46 cases $(87 \%)$ the lumen of the lymphatic channel was obliterated by tumor emboli making it difficult to assess with routine $\mathrm{H} \& \mathrm{E}$ sections. In six other cases, the comparable vessel was not present on the H\&E section. Conversely, 17 patients whose tumors showed H\&E + invasion were D2-40-. In 2/17 cases CD31 + vessels containing tumor emboli were noted corresponding to vascular invasion. In 13/17 (76\%) cases that were $\mathrm{H} \& \mathrm{E}+$ showed tumor in spaces that were D2-40 - and CD31- and thus could correspond to retraction artefact. In two cases no comparable vessel was identified on sections stained with D2-40 and CD31. 
Table 1 Association between clinicopathological characteristics and the presence of lymph vessel invasion identified by D2-40 $(N=303)$ in node-negative breast cancer patients

\begin{tabular}{|c|c|c|c|}
\hline Characteristics & No $(\%)$ & No (\%) with D2-40 +ve lymph vessel invasion & $\mathrm{P}$-value \\
\hline Age group (mean (range)) & $57.0(28-89)$ & $51.2(26-83)$ & 0.0003 \\
\hline$\leqslant 49$ & $113(37.3)$ & $44(38.9)$ & \\
\hline$\geqslant 50$ & $190(62.7)$ & $38(20.0)$ & \\
\hline \multicolumn{4}{|l|}{ Histology } \\
\hline Invasive ductal & 219 (72.3) & $67(30.6)$ & 0.06 \\
\hline Invasive lobular & $24(7.9)$ & $3(12.5)$ & \\
\hline Other & $60(19.8)$ & $12(20.0)$ & \\
\hline \multicolumn{4}{|l|}{ Tumor size } \\
\hline$<1 \mathrm{~cm}$ & $55(18.2)$ & $8(14.5)$ & 0.005 \\
\hline $1-2 \mathrm{~cm}$ & $142(46.9)$ & $35(24.7)$ & \\
\hline$>2 \mathrm{~cm}$ & $98(32.3)$ & $37(37.8)$ & \\
\hline Missing & $8(2.6)$ & $2(25)$ & \\
\hline \multicolumn{4}{|l|}{ Histologic grade (MBR) } \\
\hline 1 & $91(30.0)$ & $13(14.3)$ & 0.0001 \\
\hline 2 & 147 (48.5) & $40(27.2)$ & \\
\hline 3 & $65(21.5)$ & $29(44.6)$ & \\
\hline \multicolumn{4}{|l|}{ Nuclear grade } \\
\hline 1 & $38(12.5)$ & $6(15.8)$ & 0.007 \\
\hline 2 & $152(50.2)$ & $34(22.4)$ & \\
\hline 3 & $113(37.3)$ & $42(37.2)$ & \\
\hline \multicolumn{4}{|c|}{ Lymphovascular Invasion (H\&E) } \\
\hline No & $250(82.5)$ & $46(18.4)$ & $<0.0001$ \\
\hline Yes & $53(17.5)$ & $36(67.9)$ & \\
\hline \multicolumn{4}{|l|}{ Estrogen receptor } \\
\hline Pos & $233(76.9)$ & $52(22.3)$ & 0.005 \\
\hline Neg & $69(22.8)$ & $30(43.5)$ & \\
\hline Missing & $1(0.3)$ & 0 & \\
\hline \multicolumn{4}{|l|}{ Progesterone receptor } \\
\hline Pos & $187(61.7)$ & $44(52.4)$ & 0.09 \\
\hline Neg & 115 (38.0) & $38(33.0)$ & \\
\hline Missing & $1(0.3)$ & 0 & \\
\hline Totals & 303 & 82 (27) & \\
\hline
\end{tabular}

MBR $=$ modified bloom Richardson.

\section{Univariate Analysis and Multivariate Analysis}

Kaplan-Meier curves showed increased risk of distant recurrence and death associated with D2-40 + lymph vessel invasion (Figure $5 \mathrm{a}$ and b). Univariate analysis revealed that D2-40+ lymph vessel invasion was not associated with local recurrence $(P=0.75 ; \mathrm{RR}=1.15$; CI $0.48-2.78)$ or regional recurrence $(P=0.13 \quad \mathrm{RR}=2.83$; CI 0.69 11.6). Similarly univariate analysis of $\mathrm{H} \& \mathrm{E}+$ invasion was not associated with local $(P=0.86)$ or regional recurrence $(P=0.77)$ of breast cancer. Table 2 shows the relative risk for distant recurrence and overall survival for prognostic factors. The risk of distant recurrence was significantly increased for D2-40 + lymph vessel invasion in both univariate $(P=0.0002 ; \mathrm{RR}=3.15 ; \mathrm{CI} 1.72-5.77)$ and multivariate $(P=0.03 ; \mathrm{RR}=2.03$; CI $1.06-3.90)$ analyses (Table 2). H\&E + invasion was also a significant predictor of distant recurrence by univariate analysis $(P=0.0007$; $R R=3.03$; CI $1.60-5.74)$, but was not an independent predictor distant recurrence by multivariate analysis $(P=0.07 ; \mathrm{RR}=1.89$; CI $0.96-3.72)$. Young age $(P=0.002)$, larger tumor size $(P=0.04)$, and histological grade $(P=0.001)$ were significant predictors of distant recurrence by univariate analysis. However, age, tumor size, histological grade and ER status were not significant predictors of distant recurrence by multivariate analysis.

The relative risk for overall survival was significantly associated with D2-40 + lymph vessel invasion by univariate analyses $(P=0.0009$; $R R=2.16$; CI 1.21-3.58) but not by multivariate analysis $(P=0.12 ; \mathrm{RR}=1.65 ; \mathrm{CI} 0.88-3.10) . \mathrm{H} \& \mathrm{E}+$ invasion was also significantly associated with poor overall survival by univariate $(P=0.02$; $\mathrm{RR}=2.19$; CI $1.15-$ 4.19) but not by multivariate analysis $(P=0.21$; $\mathrm{RR}=1.55$; CI 0.78-3.10). Larger tumor size $(P=0.0009)$ and histological grade $(P=0.01)$ were 


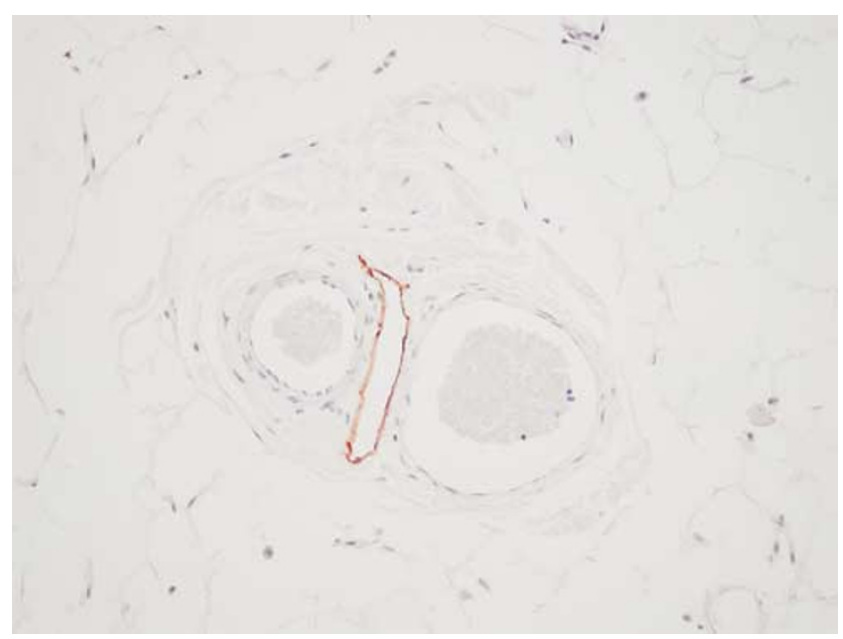

Figure 1 Lymphovascular bundle in normal breast stained with D2-40. There is positive staining of lymphatic endothelium with D2-40. The endothelium of the adjacent artery and vein are negative for D2-40 $(\times 100)$.

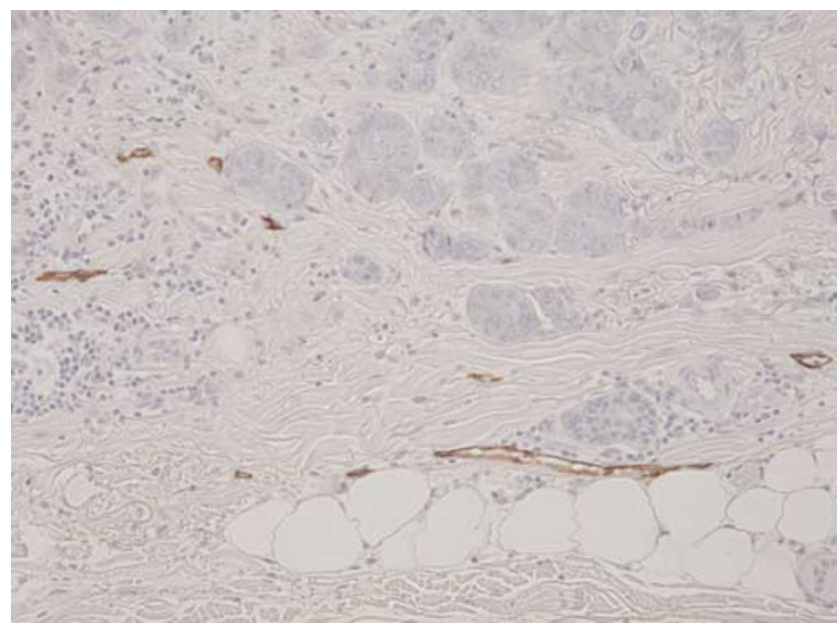

Figure 2 Invasive breast carcinoma. There is positive immunostaining of lymph vessels for D2-40 at the invasive edge of the tumor mass. No tumor emboli are noted within the lumen of the lymph vessels $(\times 100)$.

significant predictors of overall survival on univariate analysis but not by multivariate analysis. Age and ER status were not significant predictors on univariate and multivariate analysis (Table 2).

Of the 82 cases that showed D2-40 + invasion, 46 were $\mathrm{H} \& \mathrm{E}-/ \mathrm{D} 2-40+$ and 36 cases were $\mathrm{H} \& \mathrm{E}+1$ $\mathrm{D} 2-40+$. Table 3 shows the results of the subgroup analysis. The two subgroups gave similar results for the outcomes, and in the univariate analyses, both subgroups did worse than the women whose tumors were negative for lymphovascular invasion by H\&E and D2-40. Moreover, since, the confidence intervals overlapped between the two subgroups, this indicated that no strong statistical differences between the subgroups were clearly evident. Of the 221 cases

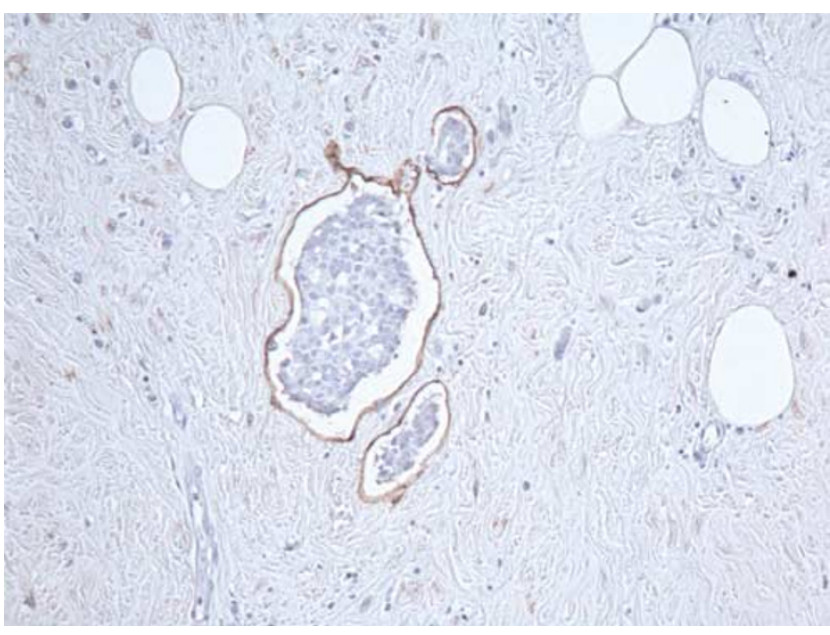

Figure 3 Invasive breast cancer. Positive staining of lymphatic endothelium with D2-40 shows the presence of tumor emboli in the lumen of the lymph vessels $(\times 100)$. These tumor emboli could be visualized on H\&E sections as they did not obliterate the lumen of the lymphatics.

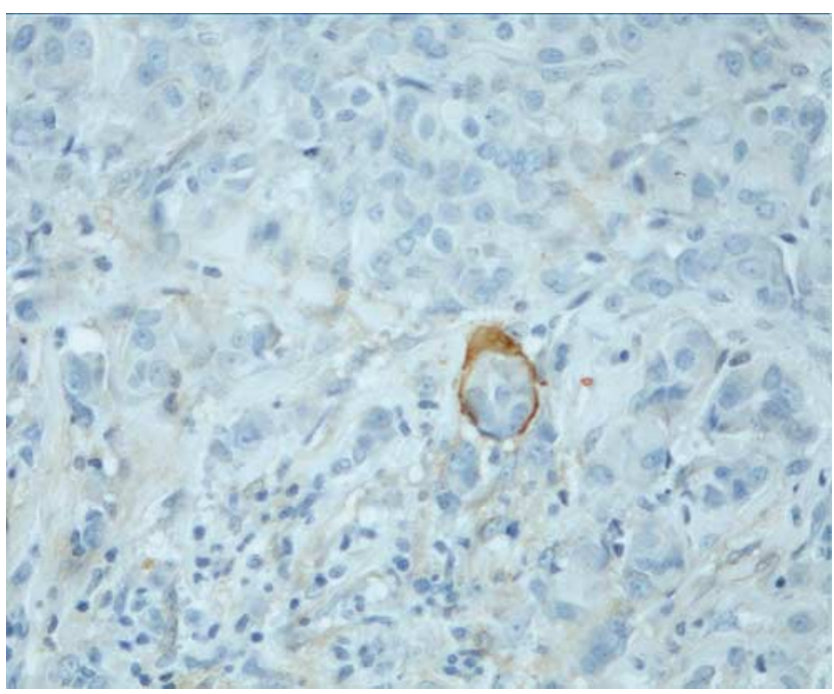

Figure 4 Invasive breast cancer. Positive staining of lymph vessel endothelium with D2-40 outlines a tumor embolus in the lumen of the lymph vessel within the tumor $(\times 200)$. This tumor embolus could not be visualized on the H\&E section as it completely obliterated the lumen.

whose tumors were D2-40- for invasion, 204 were $\mathrm{H} \& \mathrm{E}-$ whereas 17 were $\mathrm{H} \& \mathrm{E}+$. This subgroup of $\mathrm{H} \& \mathrm{E}$ +/D2-40- cases was too small to evaluate independently to assess whether these patients did as well as the patients whose tumors were H\&E-/D2-40-.

\section{Discussion}

In this study, we showed that D2-40 was positive in the endothelial cells of lymph vessels and identified tumor emboli in lymph vessels whereas D2-40 did not react with endothelium of blood vessels. This 

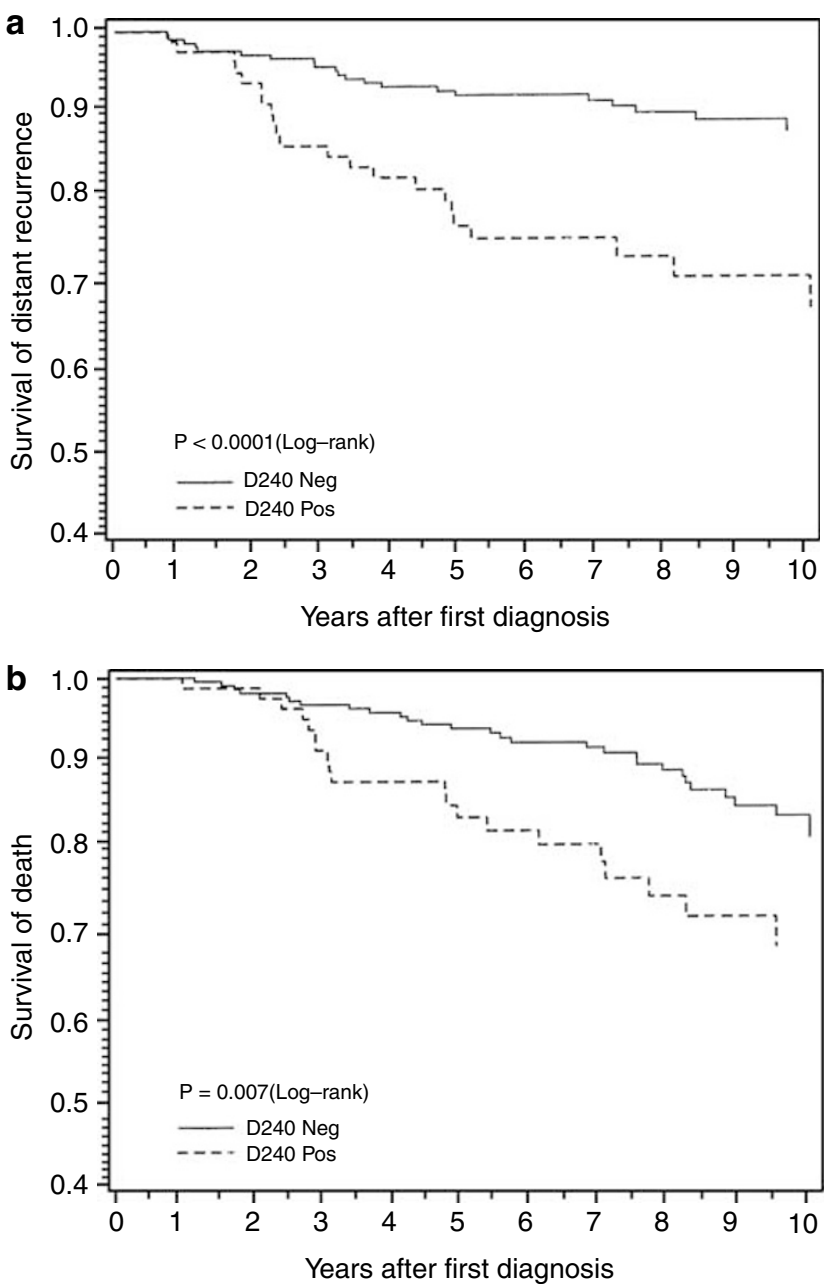

Figure 5 Kaplan-Meier curves show the association of distan recurrence and overall survival with the presence of D2-40 lymph vessel invasion. The presence of D2-40 + lymph vessel invasion is associated with increased rate of distant recurrence (a) and decreased survival (b). confirmed our previous study that showed the presence of D2-40 + lymph vessel invasion in breast cancer and other tumors. ${ }^{34,35}$ The distinction between lymph vessel and vascular invasion in breast cancer has also been demonstrated using D2-40 and the vascular marker CD34. ${ }^{36}$ Furthermore D2-40 has been shown to be the best marker for lymphatic endothelium. ${ }^{37}$

In this study, we identified lymph vessel invasion using D2-40 in $82 / 303(27 \%)$ of cases of node negative breast cancer. We showed D2-40+ lymph vessel invasion significantly correlated with $\mathrm{H} \& \mathrm{E}+$ $(P=<0.0001)$. However, $46(18.4 \%)$ patients that were negative for lymphovascular invasion on $\mathrm{H} \& \mathrm{E}$ showed D2-40 + lymph vessel invasion, indicating that the frequency of detection of lymph vessel invasion increased using D2-40. These results are similar to our pilot study whereby we showed that D2-40 increased the detection of lymphatic invasion by $20 \%$ in node negative cases and $16 \%$ in node positive cases. ${ }^{34}$ In the current study, the overall recurrence rate, which included local, regional and distant recurrences was $25 \%$ which correlates more closely with the $27 \%$ D2-40 + lymph vessel invasion when compared to the $17.5 \%$ LVI seen with H\&E. D2-40 identified tumor emboli that completely obliterated the lumen of the lymphatics. These tumor emboli could not be differentiated from nests of tumor cells on H\&E sections. The increase in sensitivity of detection of lymph vessel invasion is attributed to the demarcation of lymphatic endothelium that stains positively for D2-40 around the tumor emboli. Van Eynden's study using D2-40 also showed that lymphovascular invasion was missed on $\mathrm{H} \& \mathrm{E}$ in $20 \%$ (peritumorally) and in $65 \%$ (intratumorally) of breast cancer cases. ${ }^{36}$

The subgroup analysis of the 46 cases whose tumors were $\mathrm{H} \& \mathrm{E}-/ \mathrm{D} 2-40+$ and the 36 cases that

Table 2 Relative risk for distant recurrence and overall survival according to prognostic factors

\begin{tabular}{|c|c|c|c|c|c|c|c|c|}
\hline \multirow[t]{3}{*}{ Prognostic factor } & \multicolumn{4}{|c|}{ Distant recurrence } & \multicolumn{4}{|c|}{ Overall survival } \\
\hline & \multicolumn{2}{|c|}{ Univariate } & \multicolumn{2}{|c|}{ Multivariate } & \multicolumn{2}{|c|}{ Univariate } & \multicolumn{2}{|c|}{ Multivariate } \\
\hline & $R R(95 \% C I)$ & $\mathrm{P}$-value & $R R(95 \% C I)$ & $\mathrm{P}$-value & $R R(95 \% C I)$ & $\mathrm{P}$-value & $R R(95 \% C I)$ & $\mathrm{P}$-value \\
\hline $\begin{array}{l}\text { Lymph vessel invasion } \\
\text { D2-40+/D2-40- }\end{array}$ & $3.15(1.72-5.77)$ & 0.0002 & $2.03(1.06-3.90)$ & 0.03 & $2.16(1.21,3.58)$ & 0.009 & $1.65(0.88,3.10)$ & 0.12 \\
\hline $\mathrm{H} \& \mathrm{E}-/ \mathrm{H} \& \mathrm{E}+$ & $3.03(1.60,5.74)$ & 0.0007 & $1.89(0.96,3.72)$ & 0.07 & $2.19(1.15,4.19)$ & 0.02 & $1.55(0.78,3.10)$ & 0.21 \\
\hline Age dx (trend) & $0.96(0.93,0.98)$ & 0.002 & $0.97(0.95,1.00)$ & 0.07 & $1.00(0.97,1.02)$ & 0.72 & $1.01(0.99,1.04)$ & 0.42 \\
\hline $\begin{array}{l}\text { Tumor size } \\
\text { (trend of } 1,2,3 \text { ) }\end{array}$ & $2.03(1.26,3.27)$ & 0.004 & $1.61(0.97,2.69)$ & 0.07 & $1.81(1.16,2.80)$ & 0.009 & $1.54(0.98,2.43)$ & 0.06 \\
\hline $\begin{array}{l}\text { Histological grade } \\
\text { (trend of } 1,2,3 \text { ) }\end{array}$ & $2.07(1.33,3.22)$ & 0.001 & $1.39(0.82,2.34)$ & 0.22 & $1.69(1.12,2.55)$ & 0.01 & $1.38(0.85,2.23)$ & 0.19 \\
\hline ER status (pos/neg) & $0.55(0.29,1.05)$ & 0.07 & $0.99(0.47,2.09)$ & 0.98 & $0.59(0.32,1.10)$ & 0.10 & $0.74(0.40,1.61)$ & 0.53 \\
\hline
\end{tabular}

$\mathrm{RR}=$ relative risk, $\mathrm{CI}=$ confidence interval.

$\mathrm{RR}$ of trend of age is the risk of age at diagnosis; RR of tumor size or histological grade is the risk of lower degree to higher degree;

$\mathrm{RR}$ for other factors is the risk of pos compared to neg. 
Table 3 Associations between D2-40+ and H\&E status and specific outcomes

\begin{tabular}{|c|c|c|c|c|c|c|c|}
\hline \multirow{2}{*}{$\begin{array}{l}\text { Prognostic } \\
\text { factor } \\
\text { D2-40/H\&E }\end{array}$} & \multirow{2}{*}{$\begin{array}{c}\text { Number of } \\
\text { subjects }\end{array}$} & \multicolumn{3}{|c|}{ Distant recurrence } & \multicolumn{3}{|c|}{ Overall survival } \\
\hline & & $\begin{array}{l}\text { Number of } \\
\text { recurrences }\end{array}$ & Univariate & Multivariate & $\begin{array}{c}\text { Number of } \\
\text { deaths }\end{array}$ & Univariate & Multivariate \\
\hline Pos/Pos & 36 & 12 & $4.6(2.2-9.8)<10^{-4}$ & $2.6(1.2-5.9) 0.02$ & 10 & $2.9(1.4-6.1), 0.005$ & $2.0(0.88-4.5) 0.10$ \\
\hline Pos/Neg & 46 & 10 & $2.6(1.2-5.7) 0.02$ & $1.8(0.8-4.1) 0.15$ & 10 & $1.9(0.9-3.9), 0.10$ & $1.5(0.69-3.3) 0.30$ \\
\hline
\end{tabular}

were $\mathrm{H} \& \mathrm{E}+/ \mathrm{D} 2-40+$ showed similar results for both distant recurrence and overall survival and in the univariate analyses both subgroups did worse than the women whose tumors were negative for lymphvascular invasion by H\&E and D2-40. This suggests that $\mathrm{D} 2-40$ in addition to $\mathrm{H} \& \mathrm{E}$ would be useful in identifying lymphatic invasion in breast cancers that were $\mathrm{H} \& \mathrm{E}-$ for lymphovascular invasion.

Seventeen patients had tumors that were H\&E + for invasion were D2-40-. Possible explanations for this discrepancy could be that retraction artefact on H\&E sections causing false positives or the presence of vascular invasion, which would be CD31 + and D2-40-. As 13 of these 17 cases were CD31-, this suggests that the LVI positivity as assessed on H\&Estained sections could be due to retraction artefact. However, since two of the 17 cases were CD31+, this indicated the presence of vascular invasion. Other studies have also reported that vascular invasion is less frequent than lymphatic invasion in breast cancer. ${ }^{7,36}$

As only 17 tumors were H\&E + /D2-40- compared to the 204 cases that were H\&E-/D2-40-, subgroup analysis could not be performed owing to the small numbers of H\&E + /D2-40- cases. Nevertheless, D240 could be useful in distinguishing true lymphatic invasion from retraction artefact.

There is controversy in the literature with regard to lymphangiogenesis in breast cancer. Several studies have demonstrated the absence of lymphangiogenesis in breast cancer whereas others have reported the presence of lymphangiogenesis in breast cancer. ${ }^{38-44}$ Intratumoral lymphangiogenesis, however, has been demonstrated using LYVE-1 in some other cancers such as head and neck squamous cell carcinomas. ${ }^{45}$ The current study showed that few if any lymphatics were present within the tumor mass whereas lymphatics were present at the invasive front of the tumor as has been reported in some studies. ${ }^{40,41}$ These results support the concept that lymphangiogenesis does not occur within breast carcinoma but may occur at the invasive edge of the tumor.

There is also controversy in the literature with regard to the method whereby tumor cells gain access to lymphatics. ${ }^{39-41}$ Some researchers have postulated that the tumor invades existing lymphatics that are compressed by the tumor, since they noted very few lymphatics within the tumor mass whereas they were present at the invasive front of the tumor and furthermore the lymphatic endothelium showed no evidence of proliferative activity. $^{41,44}$ Others, however, maintain that the tumor invades new lymphatics that are formed during lymphangiogenesis within the tumor mass or at the invasive front of the tumor. ${ }^{37,39}$ In this study, most foci of D2-40 + lymph vessel invasion occurred at the invasive front of the tumor, indicating that metastasis could occur via pre-existing lymphatics or via newly formed lymphatics at the invasive front of the tumor.

$\mathrm{D} 2-40+$ lymph vessel invasion was also shown to be a significant independent predictor of distant recurrence on univariate $(P=0.0002)$ and multivariate $(P=0.03)$ analyses. When compared to all other variables, including $\mathrm{H} \& \mathrm{E}+$ lymphovascular invasion, D2-40 + lymph vessel invasion was the only variable found to be an independent predictor of distant recurrence. Recently the presence of peritumoral lymphovascular invasion has been added to the St Gallen criteria for selecting patients with operable breast cancer who would benefit from adjuvant systemic therapy. ${ }^{14}$ The peritumoral lymphovascular invasion in these guidelines is assessed on H\&E-stained sections, whereby the differentiation between vascular invasion and lymphatic invasion may not be possible and false negatives can occur. These false negatives with conventional H\&E may result in incorrect treatment decisions for patients. Thus, identification of lymph vessel invasion using D2-40 could identify a subgroup of lymph node-negative patients that may benefit from adjuvant chemotherapy.

$\mathrm{D} 2-40+$ lymph vessel invasion was also significantly associated with death $(P=0.009)$ by univariate analysis but not by the multivariate analysis $(P=0.12)$. However, as shown in Table 2, none of the other prognostic variables including age, tumor size, histological grade, and estrogen receptor status were able to predict overall survival by multivariate analysis.

Although D2-40 stains the endothelium of lymph vessels, it also reacts with the myoepithelial cells of the breast. This does not usually create a problem, as the myoepithelium is discontinuous in small ducts whereas the endothelial lining of the lymph vessels is continuous. In addition the myoepithelial cells of larger ducts are larger than the endothelial cells of lymph vessels. Occasionally stromal cells are also 
positive for D2-40. However, their morphology is different from the endothelial lining of lymph vessels.

In summary, we report that D2-40, a marker of lymphatic endothelium identifies lymph vessel invasion in breast cancers on paraffin-embedded tissues. D2-40+ lymph vessel invasion is an independent predictor of distant recurrence in node-negative breast cancer patients and is also associated with reduced survival. This marker of lymph vessel invasion can be used in addition to routine H\&E assessment of breast cancer specimens for increased accuracy of detection of lymph vessel invasion. D2-40 may also contribute significantly to the detection of lymph vessel invasion of a wide range of invasive cancers as well as to the study of lymphangiogenesis.

\section{References}

1 Fisher B, Bauer M, Wickerham DC, et al. Relation of number of positive nodes to prognosis of patients with primary breast cancer. An NSABP update. Cancer 1983;52:1551-1557.

2 Rosen PP, Saigo PE, Braun Jr DW, et al. Predictors of recurrence in stage 1 ( $\mathrm{T} 1$, No M0) breast carcinoma. Ann Surg 1981;193:15-25.

3 Roses DF, Bell DA, Flotte TJ, et al. Pathologic predictors of recurrence in Stage 1 (T1, N0 M0) breast cancer. Am J Clin Pathol 1982;78:817-820.

4 Bettelheim R, Penman HG, Thornton-Jones H, et al. Prognostic significance of peritumoral vascular invasion in breast cancer. Br J Cancer 1984;50: 771-777.

5 Bettelheim R, Munro-Neville A. Lymphatic and vascular channel involvement within infiltrative breast carcinoma as a guide to prognosis at the time of primary surgical treatment. Letter. Lancet 1981;2:631.

6 Clemente CG, Boracchi P, Andreola S, et al. Peritumural lymphatic invasion in patients with nodenegative mammary duct carcinoma. Cancer 1992;69: 1396-1403.

7 Lauria R, Perrone F, Carlomagno C, et al. The prognostic value of lymphatic and blood vessel invasion of operable breast cancer. Cancer 1995;76: 1772-1778.

8 Rosen PR, Groshen S. Factors influencing survival and prognosis in early breast cancer. (T1NOMO-T1N1MO). Surg Clin N Am 1990;70:937-962.

9 Lee AKC, DeLellis RA, Silverman ML, et al. Lymphatic and blood vessel invasion in breast carcinoma: A useful prognostic indicator? Human Pathol 1986;17: 984-987.

10 Yiangou C, Shousha S, Sinnett HD. Primary tumour characteristics and axillary lymph node status in breast cancer. Br J Cancer 1999;80:1974-1978.

11 De Laurentiis M, Gallo C, De Placido S, et al. A predictive index of axillary nodal involvement in operable breast cancer. Br J Cancer 1996;73:12411247.

12 Chua B, Ung O, Taylor $\mathrm{R}$, et al. Frequency and predictors of axillary lymph node metastases in invasive breast cancer. ANZ J Surg 2001;71:723-728.
13 Shoup M, Malinzak L, Weisenberger J, et al. Predictors of axillary lymph node metastasis in $\mathrm{T} 1$ breast carcinoma. Am Surg 1999;65:748-752.

14 Goldhirsch A, Glick JH, Gelber RD, et al. Meeting highlights: international expert consensus on the primary therapy of early breast cancer 2005. Ann Oncol 2005;16:1569-1583.

15 De Mascarel I, Bonichon F, Durand $\mathrm{M}$, et al. Obvious peritumoral emboli: An elusive prognostic factor reappraised. Multivariate analysis of 1320 node-negative breast cancers. Eur J Cancer 1998;34: 58-65.

16 Gilchrist KW, Gould VE, Hirschl S, et al. Interobserver variation in the identification of breast carcinoma in intramammary lymphatics. Hum Pathol 1982;13: 170-172.

17 Rosen PR. Tumor emboli in intramammary lymphatics in breast carcinoma: pathologic criteria for diagnosis and clinical significance. Pathol Annu 1983; 18:215-232.

18 Saigo PE, Rosen PP. The application of immunohistochemical stains to identify endothelial-lined channels in mammary carcinoma. Cancer 1987;59:51-54.

19 Bettelheim R, Mitchell D, Gusterston BA. Immunohistochemistry in the identification of vascular invasion in breast cancer. J Clin Pathol 1984;37:364-366.

20 Hanau CA, Machera H, Miettinen M. Immunohistochemical evaluation of vascular invasion in carcinomas with five different markers. App Immunohistochem 1993;1:46-50.

21 Partanen TA, Alitalo K, Miettinen M. Lack of lymphatic vascular specificity of vascular endothelial growth factor receptor3 in 185 vascular tumors. Cancer 1999;86:2406-2412.

22 Valtola R, Salven P, Heikkila P, et al. VEGFR-3 and its ligand VEGF-C are associated with angiogenesis in breast cancer. Am J Pathol 1999;154:1381-1390.

23 Jackson DG. The lymphatics revisited. New perspectives from the Hyaluronan receptor LYVE-1. Trends Cardiovasc Med 2003;13:1-7.

24 Prevo R, Banerji S, Ferguson D, et al. Mouse LYVE-1 is an endocytic receptor for hyaluronan in lymphatic endothelium. J Biol Chem 2001;276:19420-19430.

25 Mouta CC, Nasser SM, di Tomaso E, et al. LYVE-1 is not restricted to lymph vessels: expression in normal liver blood sinusoids and in human liver cancer and cirrhosis. Cancer Res 2001;61:8079-8084.

26 Wilting J, Tomarev SI, Christ B, et al. Lymphangioblasts in embryonic lymphangiogenesis. Lymphat Res Biol 2003;1:33-40.

27 Breiteneder-Geleff S, Matsui K, Soleiman A, et al. Podoplanin, a novel 43-Kd membrane protein of glomerular epithelial cells, is down-regulated in puromycin nephrosis. Am J Pathol 1997;151:1141-1152.

28 Schoppmann SF, Birner P, Studer P, et al. Lymphatic microvessel density and lymphovascular invasion assessed by anti-podoplanin immunostaining in human breast cancer. Anticancer Res 2001;21:2351-2355.

29 Birner P, Obermair A, Schindl M, et al. Selective immunohistochemical staining of blood and lymph vessels reveals independent prognostic influence of blood and lymphatic invasion in early-stage cervical cancer. Clin Cancer Res 2001;7:93-97.

30 Marks A, Sutherland DR, Bailey D, et al. Characterisation and distribution of an oncofetal antigen (M2A antigen) expressed on testicular germ tumors. Br J Cancer 1999;80:569-578. 
31 Kahn HJ, Bailey D, Marks A. Monoclonal antibody D2-40, a new marker of lymphatic endothelium, reacts with Kaposi's sarcoma and a subset of angiosarcomas. Mod Pathol 2002;15:434-440.

32 Schacht V, Dadras SS, Johnson LA, et al. Up-regulation of the lymphatic marker podoplanin, a mucin-type transmembrane glycoprotein, in human squamous cell carcinomas and germ cell tumors. Am J Pathol 2005;66:913-921.

33 Evangelou E, Kyzas PA, Trikalinos T. Comparison of the diagnostic accuracy of lymphatic endothelial markers; Bayesian approach. Mod Pathol 2005;18: 1490-1497.

34 Kahn HJ, Marks A. A new monoclonal antibody, D2-40, for detection of lymphatic invasion in primary tumors. Lab Invest 2002;82:1255-1257.

35 Niakosari F, Kahn HJ, Marks A, et al. Detection of lymphatic invasion in primary melanoma with monoclonal antibody D2-40. A new selective immunohistochemical marker of lymphatic endothelium. Arch Dermatol 2005;141:440-444.

36 Van den Eynden GG, Van der Auwera I, Van Laere SJ, et al. Distinguishing blood and lymph vessel invasion in breast cancer: a prospective immunphistochemical study. Br J Cancer 2006;94:1643-1649.

37 Van der Auwera I, Van den Eynden GG, Colpaert C, et al. Tumor lymphangiogenesis in inflammatory breast cancer: a histomorphometric study. Clin Cancer Res 2005;166:913-921.
38 Jackson DG, Prevo R, Clasper S, et al. LYVE-1, the lymphatic system and tumor lymphangiogenesis. Trends Immunol 2001;6:317-321.

39 Schoppmann SF, Bayer G, Aumayr K, , et al, and the Austrian Breast and Colorectal Cancer Study group. Prognostic value of lymphangiogenesis and lymphovascular invasion in invasive breast cancer. Ann Surg 2004;240:306-312.

40 Cunnick GH, Jiang WG, Gomez KF, et al. Lymphangiogenesis and breast cancer metastasis. Histol Histopathol 2002;17:863-870.

41 Williams CSM, Leek RD, Robson AM, et al. Absence of lymphangiogenesis and intratumoral lymph vessels in human metastatic breast cancer. J Pathol 2003;2: 195-206.

42 Vleugel MM, Bos R, van der Groep P, et al. Lack of lymphangiogenesis during breast carcinogenesis. J Clin Pathol 2004;57:746-751.

43 Choi WWL, Lewis MM, Lawson D, et al. Angiogenic and lymphangiogenic microvessel density in breast carcinoma: correlation with clinicopathologic parameters and VEGF-family gene expression. Mod Pathol 2005;18:143-152.

44 Agarwal B, Saxena R, Morimiya A, et al. Lymphangiogenesis does not occur in breast cancer. Am J Surh Pathol. 2005;29:1449-1455.

45 Beasley NJP, Prevo R, Banerji S, et al. Intratumoral lymphangiogenesis and lymph node metastases in head and neck cancer. Cancer Res 2002;62:1315-1320. 